\title{
Anwendung zeitdiskreter rekurrenter Fuzzy-Systeme zur Fehlerdiagnose
}

\author{
Fault Diagnosis employing Recurrent Fuzzy Systems
}

Andreas Schwung, Andreas Ortseifen, Jürgen Adamy, Technische Universität Darmstadt

Zusammenfassung Dieser Beitrag beschreibt die Anwendung zeitdiskreter rekurrenter Fuzzy-Systeme (DRFS) zur Fehlerdetektion und Fehlerisolation. Zum einen wird gezeigt, wie auf Basis datengestützt entworfener DRFS geeignete Residuen gebildet werden können. Zum anderen wird ein Ansatz zur Mehrfehlerisolation mit DRFS präsentiert. Dieser Ansatz basiert auf der Erweiterung klassischer statischer Fuzzy-Systeme zu DRFS. Die Anwendungsmöglichkeiten des Ansatzes werden anhand des Dreitank-Benchmarksystems illustriert. $\longrightarrow$ Summary This paper presents the application of discrete-time recurrent fuzzy systems (DRFS) for fault detection and isolation. On the one hand it is shown, how DRFS can be used for residual generation. On the other hand, an approach for isolation of multiple faults using DRFS is presented. This approach is based on an extension of static fuzzy systems for fault isolation to DRFS. The applicability of the approach is illustrated by a three tank benchmark system.

Schlagwörter Zeitdiskrete rekurrente Fuzzy-Systeme, Fehlerdetektion und Isolation, Mehrfehlerdiagnose

Keywords Discrete-time recurrent fuzzy systems, fault detection and isolation, multiple fault diagnosis

\section{Einleitung}

Mit zunehmenden Anforderungen an die Effizienz, Produktqualität und Sicherheit von industriellen Prozessen wird eine zuverlässige Überwachung und Fehlerdiagnose immer wichtiger. Diagnoseverfahren werden dazu vermehrt eingesetzt, um frühzeitig Fehler zu erkennen und dadurch größere Schäden und unnötige Kosten zu vermeiden.

Die Fehlerdiagnose läuft gewöhnlich in drei Schritten ab: Fehlerdetektion, Fehlerisolation und Fehleridentifikation. Die Detektion dient der Erkennung von Abweichungen bestimmter Prozessgrößen vom normalen Betriebszustand. Die nachfolgende Fehlerisolation stellt dann fest, welcher Fehlerfall eingetreten ist. Optional kann mithilfe der Fehleridentifikation die Größe oder das Ausmaß der aufgetretenen Fehler bestimmt werden.

Die Fehlerdetektion kann signalgestützt, beispielsweise durch Grenzwertüberwachung oder Signalanalyse, erfolgen. Zunehmend werden auch modellbasierte Ansätze verwendet, bei denen die Generierung von Residuen, die die Abweichung der Prozessgrößen von ihrem No- minalwert anzeigen, im Vordergrund steht. Es lassen sich beobachterbasierte Verfahren [7], Verfahren zur Parameterschätzung [12] und Verfahren auf Basis von Paritätsgleichungen [9] unterscheiden. Insbesondere bei Prozessen, deren analytische Modellierung schwierig ist, kommen auch Verfahren aus der künstlichen Intelligenz, zum Beispiel neuronale Netze, als Modellbildungswerkzeuge zum Einsatz [8;21].

Zur Fehlerisolation können verschiedene Arten von Klassifikatoren eingesetzt werden [17]. Eine weitere Möglichkeit sind Inferenzmethoden, die zumeist auf der Auswertung von Fehlersymptomtabellen basieren [10]. Hierbei werden vielfach statische Fuzzy-Systeme verwendet. Eine Übersicht über Verfahren zur Fehlerdetektion und -isolation (FDI) findet sich in [4] und [13].

Der vorliegende Beitrag stellt einen neuen Ansatz zur FDI vor, der auf der Verwendung von zeitdiskreten rekurrenten Fuzzy-Systemen (DRFS) basiert. DRFS wurden unabhängig voneinander in [1] und [11] aus unterschiedlichen Motiven heraus entwickelt. In [1] lag der Fokus auf der Verbindung DRFS zu Automaten, während [11] 
die Verbindung DRFS zu rekurrenten neuronalen Netzen untersuchte. Auf Basis dieser Arbeiten wurden DRFS in $[2 ; 15]$ formalisiert und systemtheoretisch untersucht. Typische Anwendungsgebiete für DRFS sind die Mustererkennung, zu der DRFS auch industriell eingesetzt werden $[1 ; 16]$, sowie die Verkehrssimulation [6].

Im Folgenden soll beleuchtet werden, in welcher Weise sich DRFS zur FDI eignen und wo ihre Vorteile liegen. Hierbei wird sowohl die modellbasierte Generierung geeigneter Residuen mithilfe lernfähiger DRFS betrachtet als auch die Fehlerisolation mittels DRFS näher vorgestellt. Letztere eignet sich insbesondere zur Isolation mehrerer kurz nacheinander auftretender Fehler.

Der Beitrag gliedert sich folgendermaßen: Abschnitt 2 stellt zunächst die Systembeschreibung zeitdiskreter rekurrenter Fuzzy-Systeme vor. In Abschnitt 3 wird zum einen die modellbasierte Generierung geeigneter $\mathrm{Re}$ siduen vorgestellt, zum anderen wird ausgehend von statischen Fuzzy-Systemen ein Ansatz zur Mehrfehlerisolation mit DRFS entwickelt. Abschnitt 4 illustriert die Methodik anhand des Dreitank-Benchmarksystems.

\section{Zeitdiskrete rekurrente Fuzzy-Systeme}

Dieser Abschnitt soll in die Systemdarstellung DRFS einführen. Weiterführende Beschreibungen finden sich in $[2 ; 14]$. Bild 1 zeigt die Struktur zeitdiskreter rekurrenter Fuzzy-Systeme. Die Funktionen $\mathbf{f}(\mathbf{x}(k), \mathbf{u}(k))$ und $\mathbf{g}(\mathbf{x}(k), \mathbf{u}(k))$ sind jeweils vollständige Fuzzy-Systeme, die aus Fuzzifizierung, Inferenz und Defuzzifizierung bestehen. Entsprechend enthalten die Eingangsvektoren $\mathbf{u}(k)$ und $\mathbf{x}(k)$ sowie die Ausgangsvektoren $\mathbf{x}(k+1)$ bzw. $\mathbf{y}(k)$ crispe Werte. Die Ausgangsfunktion $\mathbf{g}$ spielt in den weiteren Betrachtungen keine Rolle und wird deshalb nicht näher betrachtet.

Die durch die Funktion $\mathbf{f}(\mathbf{x}(k), \mathbf{u}(k))$ gegebene zeitdiskrete Dynamik wird bei DRFS linguistisch durch Regeln der Form

$\begin{array}{ll}\text { Wenn } & x_{1}(k)=L_{j_{1}}^{x_{1}} \text { und } \ldots \text { und } x_{n}(k)=L_{j_{n}}^{x_{n}} \\ \text { und } & u_{1}(k)=L_{q_{1}}^{u_{1}} \text { und } \ldots \text { und } u_{m}(k)=L_{q_{m}}^{u_{m}}, \\ \text { dann } & x_{1}(k+1)=L_{w_{1}}^{x_{1}} \text { und ... und } x_{n}(k+1)=L_{w_{n}}^{x_{n}}\end{array}$

beschrieben. Hierbei sind $L_{j_{i}}^{x_{i}}, L_{q_{p}}^{u_{p}}$ und $L_{w_{i}}^{x_{i}}$ die linguistischen Werte der linguistischen Zustände $x_{i}(k)$ im Zeitschritt $k$, der linguistischen Eingänge $u_{p}(k)$ und der linguistischen Zustände $x_{i}(k+1)$ im folgenden Zeitschritt $k+1$.

Für ein System mit zwei Zuständen, die jeweils die linguistischen Werte $L_{1}^{x_{i}}=$ „niedrig“, $L_{2}^{x_{i}}=$ „mittel“ und

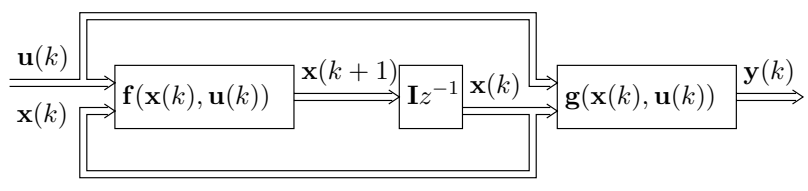

Bild 1 Blockschaltbild eines zeitdiskreten rekurrenten Fuzzy-Systems.
$L_{3}^{x_{i}}=$,hoch“ besitzen, und einem Eingang mit den linguistischen Werten $L_{1}^{u}=$,negativ“ und $L_{2}^{u}=$ „positiv" lässt sich beispielsweise die folgende Regel

Wenn $x_{1}(k)=$,hoch“ und $x_{2}(k)=$,mittel“

und $u(k)=$,negativ“,

dann $\quad x_{1}(k+1)=$,niedrig“ und $x_{2}(k+1)=$ „mittel

angeben. Durch die Angabe weiterer Regeln obiger Form entsteht schließlich eine vollständige Regelbasis.

Fasst man obige linguistische Werte jeweils in gemeinsamen linguistischen Vektoren $\mathbf{L}_{\mathbf{j}}^{\mathbf{x}}, \mathbf{L}_{\mathbf{q}}^{\mathbf{u}}$ und $\mathbf{L}_{\mathbf{w}}^{\mathbf{x}}$ zusammen, so ergibt sich die folgende äquivalente Kurzform der Regeln (1)

Wenn $\quad \mathbf{x}(k)=\mathbf{L}_{\mathbf{j}}^{\mathbf{x}}$ und $\mathbf{u}(k)=\mathbf{L}_{\mathbf{q}}^{\mathbf{u}}$,

dann $\quad \mathbf{x}(k+1)=\mathbf{L}_{\mathbf{w}}^{\mathbf{x}}$

in Form einer linguistischen Differenzengleichung mit $\mathbf{x} \in$ $\mathbb{R}^{n}, \mathbf{u} \in \mathbb{R}^{m}$.

Um aus dieser linguistischen Differenzengleichung einen mathematischen Ausdruck zu gewinnen, wird jedem linguistischen Wert $L_{j_{i}}^{x_{i}}$ jeder Zustandsvariablen $x_{i}(k)$ auf dem Intervall $X_{i}$ eine Zugehörigkeitsfunktion $\mu_{j_{i}}^{x_{i}}\left(x_{i}\right)$ zugeordnet. Ebenso werden Zugehörigkeitsfunktionen $\mu_{q_{p}}^{u_{p}}\left(u_{p}\right)$ für die Eingangsgrößen $u_{p}(k)$ eingeführt. Die Abszisse des Maximalwerts der Zugehörigkeitsfunktion $s_{j_{i}}^{x_{i}}$ bzw. $s_{q_{p}}^{u_{p}}$ wird dabei als Kernposition bezeichnet. Als Zugehörigkeitsfunktionen der Zustände im Zeitschritt $x_{i}(k+1)$ werden Singletons $s_{w_{i}}^{x_{i}}$ verwendet, die auch als Ausgangskernpositionen bezeichnet werden. Ähnlich wie die linguistischen Werte lassen sich auch die Kernpositionen in Vektorform $\mathbf{s}_{\mathbf{j}}^{\mathbf{x}}, \mathbf{s}_{\mathbf{q}}^{\mathbf{u}}$ und $\mathbf{s}_{\mathbf{w}}^{\mathbf{x}}$ zusammenstellen.

Die Zugehörigkeitsfunktionen sollen außerdem die folgenden Bedingungen erfüllen:

1. Beschränkung: $\mu_{j_{i}}^{x_{i}}\left(x_{i}\right) \in[0,1]$ für alle $x_{i} \in X_{i}$,

2. Konvexität: $\left\{\begin{array}{l}\mu_{j_{i}}^{x_{i}}\left(x_{i}\right) \text { monoton steigend } \forall x_{i}<s_{j_{i}}^{x_{i}} \\ \mu_{j_{i}}^{x_{i}}\left(x_{i}\right) \text { monoton fallend } \forall x_{i}>s_{j_{i}}^{x_{i}}\end{array}\right.$

3. Partitionierung: $\sum_{j_{i}} \mu_{j_{i}}^{x_{i}}\left(x_{i}\right)=1$ für alle $x_{i} \in X_{i}$ und $\mu_{j_{i}}^{x_{i}}\left(s_{j_{i}}^{x_{i}}\right)=1$ und $\mu_{j_{i}}^{x_{i}}\left(s_{l_{i}}^{x_{i}}\right)=0$ für $j_{i} \neq l_{i}$,

4. Stetigkeit: $\mu_{j_{i}}^{x_{i}}\left(x_{i}\right)$ ist stetig in $X_{i}$.

Verwendet man nun Dreieck- und Rampenfunktionen als Zugehörigkeitsfunktionen, dann lässt sich das DRFS vollständig durch die Lage der Kernpositionen beschreiben (Bild 2a oben).

Als Operatoren für die Aggregation und Implikation wird das algebraische Produkt, für die Akkumulation die einfache Summe verwendet. Die Defuzzifizierung erfolgt mit der Center-of-Singletons-Methode. Mit den Bedingungen 1.-4. und gemäß [2] ergibt sich schließlich die mathematische Beschreibung der Dynamik zu

$\mathbf{x}(k+1)=\sum_{\mathbf{j}, \mathbf{q}} \mathbf{s}_{\mathbf{w}(\mathbf{j}, \mathbf{q})}^{\mathbf{x}} \prod_{i=1}^{n} \mu_{j_{i}}^{x_{i}}\left(x_{i}(k)\right) \prod_{p=1}^{m} \mu_{q_{p}}^{u_{p}}\left(u_{p}(k)\right)$. 
a)

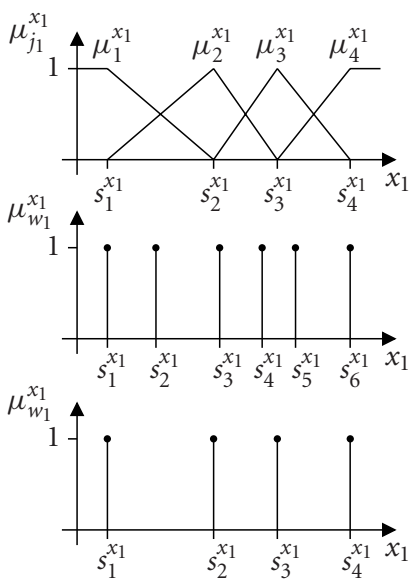

b)

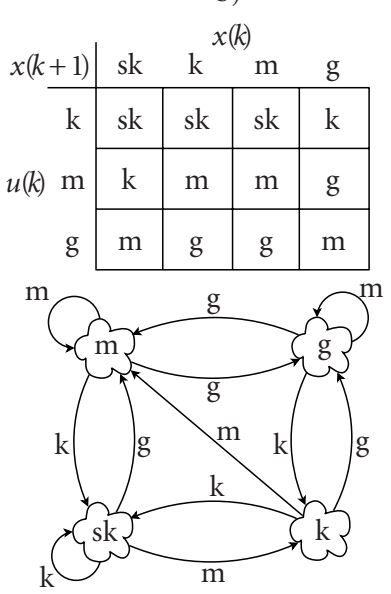

Bild 2 (a) Zugehörigkeitsfunktionen mit entsprechenden Kernpositionen $s_{j_{1}}^{x_{1}}$ (oben) sowie Ausgangskernpositionen $s_{w_{1}}^{x_{1}}$ für ein DRFS ohne (mitte) und mit (unten) Rückkopplungskorrespondenz. (b) Zustandsgraph für eine Regelbasis mit einer Zustands- und Eingangsgröße. Die welligen Knoten symbolisieren die unscharfen Zustände [2].

Die Ausgangskernpositionen $s_{w_{i}}^{x_{i}}$ können zunächst beliebige Werte aus dem Intervall $X_{i}$ annehmen (vgl. Bild 2a mittlere Abbildung). Dies hat bei der Modellierung zeitdiskreter dynamischer Systeme durch DRFS Vorteile, da mehr Parameter und damit mehr Freiheitsgrade zur Verfügung stehen. So definierte DRFS werden in Abschnitt 3.2 zur modellbasierten Residuengenerierung eingesetzt.

Bei der Anwendung DRFS zur Fehlerisolation wird dagegen die folgende zusätzliche Bedingung an die Definition der Zugehörigkeitsfunktionen der Zustände im Zeitschritt $k+1$ gestellt:

5. Rückkopplungskorrespondenz: Sei $\mathcal{S}_{i}$ die Menge, die alle Kernpositionen $s_{j_{i}}^{x_{i}}$ des Zustands $x_{i}(k)$ enthält. Dann gilt für die Ausgangszugehörigkeitsfunktionen: $s_{w_{i}}^{x_{i}} \in \mathcal{S}_{i}$ für alle $w_{i}=1, \ldots, \zeta(i)$ und $i=1, \ldots, n$, wobei $\zeta(i)$ die Zahl an Kernpositionen des Zustands $x_{i}$ bezeichnet.

Anschaulich bedeutet dies, dass die Lage der Ausgangskernpositionen $s_{w_{i}}^{x_{i}}$ des Zustands $x_{i}(k+1)$ mit den Kernpositionen der Eingangszugehörigkeitsfunktion $s_{j_{i}}^{x_{i}}$ des Zustands $x_{i}(k)$ identisch ist (vgl. Bild 2a unten).

Mit der Rückkopplungskorrespondenz ist das DRFS nun folgendermaßen interpretierbar [2]: Operiert das DRFS ausschließlich auf den Kernpositionen, so kann es als linguistischer Zustandsautomat aufgefasst werden. Entsprechend kann für die Operation auf den Kernpositionsvektoren auch ein Zustandsgraph angegeben werden, der das Verhalten des DRFS an den Kernpositionen qualitativ beschreibt. Bild $2 \mathrm{~b}$ zeigt dies für eine einfache Regelbasis. Die Kernpositionen der $\mathrm{Zu}$ stände bilden die Zustände des Graphen. Die Regelbasis definiert dann, welche Transitionen bei welcher Kombination von Kernpositionen der Eingänge möglich sind. $\mathrm{Zu}$ beachten ist, dass im Gegensatz zu Zustandsautomaten [18] DRFS auch beliebige Werte zwischen den
Kernpositionen annehmen können, wenn das DRFS nicht auf Kernpositionen operiert. Es ergibt sich insgesamt ein zeitdiskretes, aber im Gegensatz zu Zustandsautomaten nicht wertediskretes System.

\section{Fehlerdiagnose mit rekurrenten Fuzzy-Systemen}

Dieser Abschnitt soll näher beleuchten, wie die im vorigen Abschnitt vorgestellten zeitdiskreten rekurrenten FuzzySysteme zur Fehlerdiagnose eingesetzt werden können. Im ersten Schritt wird die Eignung DRFS zur Fehlerdetektion betrachtet und gezeigt, wie passende Residuen aus trainierten DRFS gewonnen werden können. Im zweiten Schritt wird dann die Verwendung DRFS zur Fehlerisolation gezeigt. Dieser Ansatz stellt eine Erweiterung statischer Fuzzy-Systeme zur Fehlerisolation dar und eignet sich insbesondere zur Isolation mehrerer nacheinander auftretender Fehler.

\subsection{Modellbildung}

Von zentraler Bedeutung für die modellgestützte Fehlerdetektion ist die Generierung geeigneter Residuen, die den Status des Prozesses beschreiben und somit Abweichungen vom fehlerfreien Systemzustand anzeigen. Hierzu benötigt man ein genaues Modell des Systems, das mithilfe einer physikalischen Modellbildung bestimmt werden kann. Allerdings hat man es des Öfteren auch mit Prozessen zu tun, bei denen eine physikalische Modellbildung nicht oder kaum möglich ist. Dann werden datengestützte Verfahren wie neuronale Netze zur Modellbildung verwendet. $\mathrm{Zu}$ dieser Klasse von Verfahren gehören auch DRFS, bei denen die datengestützte Modellbildung durch qualitatives Prozesswissen ergänzt werden kann.

Zur Modellbildung mit DRFS können ähnlich wie bei neuronalen Netzen Lernalgorithmen eingesetzt werden. Ein solcher Ansatz wurde in [3] für zeitkontinuierliche rekurrente Fuzzy-Systeme vorgestellt und wird im Folgenden auf DRFS übertragen. Ausgangspunkt des Verfahrens sind $N$ Messdatensätze

$\left[\mathbf{u}^{(v)}(k), \mathbf{x}^{(v)}(k), \mathbf{x}^{(v)}(k+1)\right], \quad v=1, \ldots, N$,

mit denen das DRFS an das zu modellierende System angepasst werden kann. Diese Anpassung kann durch eine Optimierung der Kernpositionen erfolgen, wobei die Summe der quadratischen Fehler zwischen gemessenem und durch das DRFS geschätzten Zustandswert

$J_{i}=\sum_{v=1}^{N}\left(\hat{x}_{i}^{(v)}(k+1)-x_{i}^{(v)}(k+1)\right)^{2}$

minimiert wird. Bei der Optimierung kann zwischen den beiden Parametergruppen Ausgangskernpositionen $s_{w_{i}}^{x_{i}}$ und Kernpositionen der Eingangszugehörigkeitsfunktionen $s_{j_{i}}^{x_{i}}$ und $s_{q_{p}}^{u_{p}}$ unterschieden werden.

Die Optimierung der Ausgangskernpositionen mittels des Gütekriteriums (6) stellt ein Least-Square Problem 
dar. Um dies zu zeigen, wird die $i$-te Komponente der Gl. (4) wie folgt umgestellt:

$$
\begin{aligned}
x_{i}(k+1) & =\sum_{\mathbf{j}, \mathbf{q}} s_{w_{i}(\mathbf{j}, \mathbf{q})}^{x_{i}} \prod_{l=1}^{n} \mu_{j_{l}}^{x_{l}}\left(x_{l}\right) \prod_{p=1}^{m} \mu_{q_{p}}^{u_{p}}\left(u_{p}\right) \\
& =\sum_{\mathbf{j}, \mathbf{q}} \boldsymbol{\Xi}_{(\mathbf{j}, \mathbf{q})}(\mathbf{x}, \mathbf{u}) \cdot s_{w_{i}(\mathbf{j}, \mathbf{q})}^{x_{i}}=\mathbf{\Xi}^{T}(\mathbf{x}, \mathbf{u}) \cdot \boldsymbol{\theta}_{i},
\end{aligned}
$$

wobei im letzten Schritt die Summe über alle Regeln durch das Vektorprodukt dargestellt wurde. Der Vektor $\boldsymbol{\theta}_{i}$ enthält dabei alle Ausgangskernpositionen $s_{w_{i}}^{x_{i}}$ des Zustands $x_{i}$, während im Vektor $\boldsymbol{\Xi}(\mathbf{x}, \mathbf{u})$ die Prämissen aller Regeln zusammengefasst sind. Setzt man nun (7) in (6) ein, so ergibt sich mit der Matrix $\Psi$ und den gemessenen Vektoren $\mathbf{z}_{i}$

$\boldsymbol{\Psi}=\left(\begin{array}{c}\boldsymbol{\Xi}^{T}\left(\hat{\mathbf{x}}^{(1)}, \hat{\mathbf{u}}^{(1)}\right) \\ \vdots \\ \boldsymbol{\Xi}^{T}\left(\hat{\mathbf{x}}^{(N)}, \hat{\mathbf{u}}^{(N)}\right)\end{array}\right), \mathbf{z}_{i}=\left(\begin{array}{c}\hat{\dot{x}}_{i}^{(1)} \\ \vdots \\ \hat{\dot{x}}_{i}^{(N)}\end{array}\right)$

das quadratische Optimierungsproblem

$J_{i}\left(\boldsymbol{\theta}_{i}\right)=\left(\mathbf{z}_{i}-\boldsymbol{\Psi} \cdot \boldsymbol{\theta}_{i}\right)^{T}\left(\mathbf{z}_{i}-\boldsymbol{\Psi} \cdot \boldsymbol{\theta}_{i}\right) \rightarrow \min$.

Dessen Lösung in der $i$-ten Komponente lautet

$\boldsymbol{\theta}_{i}=\left[\boldsymbol{\Psi}^{T} \cdot \boldsymbol{\Psi}\right]^{-1} \boldsymbol{\Psi}^{T} \mathbf{z}_{i}$.

Es ergeben sich insgesamt also $i=1, \ldots, n$ konvexe Optimierungsprobleme für die Ausgangskernpositionen.

Die Optimierung der Kernpositionen der Eingangszugehörigkeitsfunktionen $\mathbf{s}_{\mathbf{j}}^{\mathbf{x}}, \mathbf{s}_{\mathbf{q}}^{\mathbf{u}}$ ist dagegen erheblich schwieriger, da die Eingangskernpositionen $s_{j_{l}}^{x_{l}}$ gemäß

$\mu_{j l}^{x_{l}}\left(x_{l}\right)=\left\{\begin{array}{l}\frac{x_{l}-s_{j_{l}-1}^{x_{l}}}{s_{j_{l}}-s_{j_{l}-1}^{x_{l}}}, \text { wenn } s_{j_{l}-1}^{x_{l}}<x_{l}<s_{j_{l}}^{x_{l}}, \\ \frac{s_{j_{l}+1}^{x_{l}}-x_{l}}{s_{j_{l}+1}^{x_{l}}-s_{j_{l}}^{x_{l}}}, \quad \text { wenn } s_{j_{l}}^{x_{l}}<x_{l}<s_{j_{l}+1}^{x_{l}}, \\ 0, \text { sonst }\end{array}\right.$

nichtlinear in die Zugehörigkeitsfunktion eingehen. Verwendet man wiederum Gütekriterium (6) und setzt Gl. (7) ein, so ergibt sich ein nichtkonvexes Optimierungsproblem, $\mathrm{zu}$ dessen Lösung in [3] ein Gradientenverfahren vorgeschlagen wird.

Die Optimierung beider Parametergruppen erfolgt nun iterativ, d.h., es wird immer abwechselnd ein Iterationsschritt des Gradientenverfahrens und eine LeastSquare Optimierung durchgeführt, bis die Änderung der Parameter eine vorgegebene Grenze unterschreitet. Das datengestützt optimierte DRFS kann damit ein technisches System sehr genau modellieren.

\subsection{Residuengenerierung}

Es stellt sich nun die Frage, wie auf Basis eines solchen Modells geeignete Residuen gebildet werden können, mit denen sich Fehler detektieren lassen. Da DRFS nichtlineare Zustandsraummodelle des Systems darstellen, bieten sich als Residuen $\mathbf{r}$ insbesondere die Differenz zwischen gemessenen $\mathbf{x}$ und durch das DRFS geschätzten Systemzuständen $\hat{\mathbf{x}}$

$\mathbf{r}=\mathbf{x}-\hat{\mathbf{x}}$

an. Entsprechend gehen wir im Folgenden davon aus, dass die Zustände des Systems gemessen werden können. Eine Erweiterung dahingehend, dass ausschließlich Ausgangsgrößen zur Residuengenerierung verwendet werden ist allerdings auch möglich. In diesem Fall muss neben der Dynamikfunktion $\mathbf{f}(\mathbf{x}, \mathbf{u})$ auch die Ausgangsfunktion $\mathbf{g}(\mathbf{x}, \mathbf{u})$ datenbasiert optimiert werden.

Die einfachste Möglichkeit, Residuen zu generieren, ist nun, das DRFS dem realen Prozess parallel zu schalten. Tritt dann ein Fehler im Prozess auf, stimmen die Zustände $x_{i}$ des fehlerhaften Prozesses nicht mehr mit den Zuständen $\hat{x}_{i}$ des DRFS überein und $r_{i}$ weicht von null ab. Allerdings führt dieser einfache Ansatz dazu, dass sich beim Auftreten eines Fehlers im Allgemeinen alle Residuen $r_{i}$ verändern. Wünschenswert für eine gute Fehlerisolation ist dagegen, dass ein Residuum nur auf einige wenige Fehler reagiert, also nur auf wenige Fehler sensitiv ist. Dies erleichtert dann die Fehlerisolation.

Deshalb wird eine veränderte Anordnung von DRFS zur Fehlerdetektion verwendet, die Bild 3 zeigt. Im Gegensatz zur bekannten Struktur DRFS (Bild 1) wird in dieser Anordnung nur noch eine Zustandsgröße $\hat{x}_{i} \mathrm{zu}$ rückgeführt. Für die anderen Zustände $\mathbf{x}$ werden die Messgrößen als zusätzliche Eingänge verwendet. Diese Anordnung hat den Vorteil, dass das so gebildete Residuum nur noch sensitiv auf Fehler ist, die direkt auf die rückgeführte Zustandsgröße wirken. Dagegen ist das Residuum durch die zusätzlich gemessenen restlichen $\mathrm{Zu}$ standsgrößen im Gegensatz zur reinen Parallelschaltung nicht sensitiv gegenüber Fehlern, die andere Zustandsgrößen beeinflussen.

Ein so aufgebautes DRFS wird nun für jede $\mathrm{Zu}$ standsgröße eingesetzt, wobei als Residuen wiederum die Differenzen der gemessenen und geschätzten Zustände verwendet werden. Diese Residuen werden im Symptomgenerator mit den Residuen im fehlerfreien Fall $\mathbf{r}_{0}=\mathbf{0}$ verglichen. Optional kann diese Größe auch noch umgerechnet werden, beispielsweise durch eine Normierung auf den Eingangswert, um die Abhängigkeit vom Anregungssignal zu verringern. Als Ausgang ergibt sich der

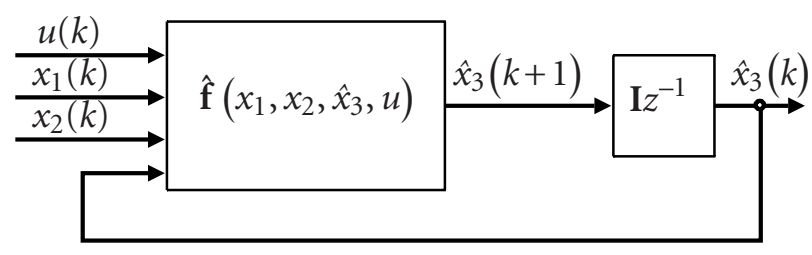

Bild 3 Beispielhafte Verschaltung eines zeitdiskreten rekurrenten FuzzySystems mit drei Zuständen und einem Eingang zur Fehlerdetektion. 


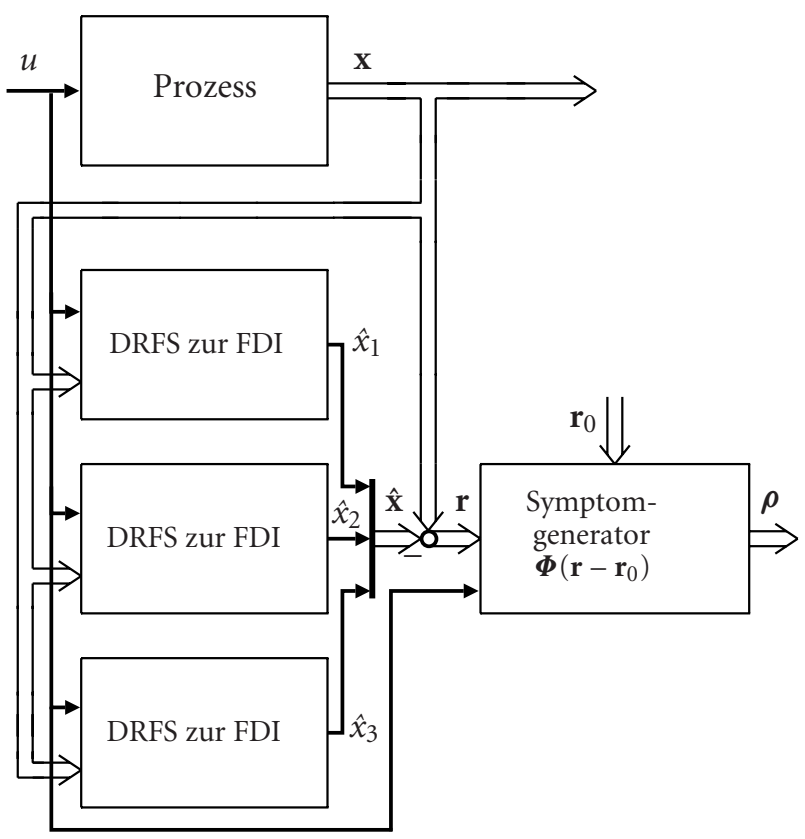

Bild 4 Struktur der Fehlerdetektion mit zeitdiskreten rekurrenten FuzzySystemen für ein System mit drei Zuständen und einem Eingang.

Fehlersymptomvektor als Funktion $\boldsymbol{\Phi}$ der Residuen $\mathbf{r}$ und $\mathbf{r}_{0}$

$\rho=\Phi\left(\mathbf{r}, \mathbf{r}_{0}\right)=\boldsymbol{\Phi}\left(\mathbf{r}-\mathbf{r}_{0}\right)$.

Die resultierende Struktur zur Fehlerdetektion ist in Bild 4 gezeigt. Wichtig zu bemerken ist, dass immer dasselbe DRFS verwendet wird. Entsprechend muss nur ein Modell des Prozesses datengestützt gebildet werden. Die DRFS unterscheiden sich lediglich in der Verschaltung, d. h. in der Zustandsgröße, die zurückgeführt wird. Auf Basis dieser Struktur werden nun modellgestützt Residuen und daraus Symptome generiert. Wie diese Symptome zur Fehlerisolation eingesetzt werden können, wird im nächsten Abschnitt erläutert.

\subsection{Mehrfehlerisolation}

Viele Verfahren zur FDI konzentrieren sich auf die Diagnose von einzeln auftretenden Fehlern. Dies setzt voraus, dass nach Auftreten eines Fehlers dieser schnellstmöglich behoben wird, da die Fehlerdiagnose ansonsten keine verlässlichen Ergebnisse liefert. Eine schnelle Reparatur ist aber oftmals nicht möglich oder auch nicht erforderlich, insbesondere wenn die Fehler keinen kritischen Prozesszustand hervorrufen. In solchen Fällen können also bis zur Wartung mehrere Fehler auftreten, die detektiert und isoliert werden sollen [5;19]. Hierzu wird ein Ansatz mit DRFS entwickelt.

Zur Herleitung der Methodik wird im ersten Schritt ein statisches Fuzzy-System (SFS) zur Fehlerisolation vorgestellt. Dieses SFS stößt allerdings, wie sich zeigen wird, im Mehrfehlerfall an seine Grenzen. Deshalb wird im zweiten Schritt eine Erweiterung auf DRFS vorgestellt, mit dem dann auch mehrere nacheinander auftretende Fehler parallel isoliert werden können.

\section{Fehlerisolation mit statischen Fuzzy-Systemen}

Der Einsatz statischer Fuzzy-Systeme zur Fehlerisolation ist ein bekannter und vielfach verwendeter Ansatz [13]. Die Auslegung des Fuzzy-Systems geschieht hierbei auf Basis einer Fehlersymptomtabelle, wie in Tabelle $1 \mathrm{zu}$ sehen. Diese Tabelle zeigt die Symptome beim Auftreten eines bzw. mehrerer Fehler. Die Symptome können hierbei positiv, null oder negativ werden. Auf Basis dieser Tabelle kann dann durch Regeln der Form

Wenn $\quad \rho_{1}=L_{q_{1}}^{\rho_{1}}$ und $\ldots$ und $\rho_{m}=L_{q_{m}}^{\rho_{m}}$,

dann $\varphi_{1}=L_{w_{1}}^{\varphi_{1}}$ und $\ldots$ und $\varphi_{l}=L_{w_{l}}^{\varphi_{l}}$

auf den Fehler geschlossen werden. Hierbei ist $L_{q_{v}}^{\rho_{v}}$ der linguistische Wert der Abweichung des Symptoms $\rho_{v}$ vom Nominalwert und $L_{w_{r}}^{\varphi_{r}}$ der Zugehörigkeitswert, dass der Fehlerfall $\varphi_{r}$ eingetreten ist. Im Regelfall werden für die Symptome jeweils drei linguistische Werte $L_{1_{v}}^{\rho_{v}}=$ „negativ“, $L_{2_{v}}^{\rho_{v}}=$ „null“, $L_{3_{v}}^{\rho_{v}}=$ „positiv“ verwendet. Die beiden linguistischen Werte $L_{1_{r}}^{\varphi_{r}}=$ „kein Fehler“ und $L_{2_{r}}^{\varphi_{r}}=$ „Fehler“ beschreiben die Fehlerzugehörigkeit.

Die Möglichkeit, Fehler zu isolieren, hängt von den gewählten Symptomen ab. Dies soll am Beispiel der Fehlersymptomtabelle 1 verdeutlicht werden. Offensichtlich sind die Einzelfehler vollständig isolierbar, da eine eindeutige Zuordnung von Symptomen zu Fehlerfällen möglich ist. Betrachtet man allerdings zusätzlich auch die Doppelfehler $\left(\varphi_{i}, \varphi_{j}\right)$, im Weiteren als $\varphi_{i j}$ bezeichnet, so ist keine eindeutige Zuordnung mehr möglich. Die Fehlerfälle $\varphi_{2}, \varphi_{32}, \varphi_{23}$ sowie $\varphi_{3}, \varphi_{21}, \varphi_{12}$ weisen jeweils dieselben Symptome auf und sind somit nicht unterscheidbar.

Es zeigt sich also, dass das SFS insbesondere im Mehrfehlerfall die Fehler nicht mehr eindeutig isolieren kann. Unter der Voraussetzung, dass die Fehler nacheinander auftreten, kann allerdings mithilfe einer Erweiterung SFS auf DRFS die Zahl der isolierbaren Fehler deutlich erhöht werden, wie im Weiteren demonstriert wird.

\section{Fehlerisolation mit zeitdiskreten rekurrenten Fuzzy-Systemen}

Der vorherige Abschnitt hat gezeigt, dass die Mehrfehlerisolation mit SFS selbst bei Symptomtabellen, die eine Isolation aller Einzelfehler erlauben, meist nicht möglich ist. Bezieht man dagegen Informationen über die dynamische Entwicklung der Symptome mit ein, so kann die

Tabelle 1 Fehlersymptomtabelle mit drei Symptomen $\rho_{p}$ für drei Fehlerfälle $\varphi_{i}$ und die zugehörigen Doppelfehler $\varphi_{i j}$. Hierbei bezeichnet $i$ den ersten, $j$ den zweiten aufgetretenen Fehler sowie $\varphi_{0}$ den fehlerfreien Zustand. Die Symptome können positiv (+), negativ (-) oder null (0) werden.

\begin{tabular}{cccccccc}
\hline & $\varphi_{0}$ & $\varphi_{1}$ & $\varphi_{2}$ & $\varphi_{3}$ & $\varphi_{12}, \varphi_{21}$ & $\varphi_{13}, \varphi_{31}$ & $\varphi_{23}, \varphi_{32}$ \\
\hline$\rho_{1}$ & 0 & 0 & + & + & + & + & + \\
$\rho_{2}$ & 0 & - & 0 & - & - & - & 0 \\
$\rho_{3}$ & 0 & - & 0 & 0 & 0 & - & 0 \\
\hline
\end{tabular}


Mehrfehlerisolation deutlich verbessert werden. Dies wird durch die Erweiterung SFS zu DRFS ermöglicht.

Hierbei werden die Fehlerfälle $\varphi_{r}$ nicht mehr als Ausgänge eines SFS, sondern als Zustände des DRFS aufgefasst. Durch die Rückführung dieser Zustände, also der Fehlerzugehörigkeiten, werden dann die neuen Fehlerzugehörigkeiten auf Basis der Residuen und der alten Fehlerzugehörigkeiten bestimmt. Es ergeben sich nun Regeln der Form

$$
\begin{array}{ll}
\text { Wenn } & \varphi_{1}(k)=L_{j_{1}}^{\varphi_{1}} \text { und } \ldots \text { und } \varphi_{l}(k)=L_{j_{l}}^{\varphi_{l}} \\
\text { und } & \rho_{1}(k)=L_{q_{1}}^{\rho_{1}} \text { und } \ldots \text { und } \rho_{m}(k)=L_{q_{m}}^{\rho_{m}}, \\
\text { dann } & \varphi_{1}(k+1)=L_{w_{1}}^{\varphi_{1}} \text { und } \ldots \text { und } \varphi_{l}(k+1)=L_{w_{l}}^{\varphi_{l}} .
\end{array}
$$

Das zugrundeliegende Fuzzy-System ist dabei ein DRFS, bei dem die Rückkopplungskorrespondenz nach Abschnitt 2 erfüllt ist.

Betrachtet man wieder die Fehlersymptomtabelle 1, so ergibt sich nun eine deutlich verbesserte Isolation der Mehrfehlerfälle. Hierzu betrachte man beispielhaft den Mehrfehlerfall $\varphi_{12}$, der mithilfe des SFS nicht vom Einzelfehler $\varphi_{3}$ unterschieden werden kann. Ist bereits Fehler $\varphi_{1}$ aufgetreten und korrekt isoliert worden, so wird diese Information rückgeführt. Bei einer Änderung der Symptome auf „+-0“ und der zusätzlichen Information, dass der Fehler $\varphi_{1}$ bereits erkannt wurde, kann dann auf den Doppelfehler $\varphi_{12}$ geschlossen werden. Der Einzelfehler $\varphi_{3}$ ist so ausgeschlossen. Ähnliches geschieht, wenn zuerst Fehler $\varphi_{2}$ auftritt. Ein Sonderfall sind die Doppelfehler $\varphi_{23}$ bzw. $\varphi_{32}$. Offensichtlich hängt die Isolierbarkeit von der Reihenfolge ab, in der die Fehler auftreten. Tritt zuerst $\varphi_{3}$ mit den Symptomen „+-0“ auf, ist die Isolation des Doppelfehlers $\varphi_{32}$ durch die Änderung der Symptome auf „+ +00 “ möglich. Tritt dagegen zuerst $\varphi_{2}$ mit den Symptomen „+ 00“ auf, so kann der Doppelfehler $\varphi_{23}$ mit den gleichen Symptomen „+ 00 “ nicht erkannt werden, da sich die Symptome nicht verändert haben. Insgesamt kann die Zahl der isolierbaren Fehler durch die Erweiterung auf DRFS deutlich erhöht werden.

Ein Nachteil bei der Verwendung DRFS ist die im Vergleich zu SFS deutlich höhere Zahl an Regeln, die eingestellt werden müssen. Da die DRFS zur Fehlerisolation die Rückkopplungskorrespondenz aufweisen, besteht allerdings aufgrund der Nähe zu Zustandsautomaten die Möglichkeit, sich zur Auslegung der Fehlerisolation an einem solchen Zustandsautomaten zu orientieren. Sieht man die Fehler als Zustandsgrößen des DRFS an, kann man deren Kernpositionen als Zustände eines Automaten auffassen. Die Regelbasis definiert dann, ob und welcher Zustandsübergang bei bestimmten Symptomen eintritt. Einen Ausschnitt des sich aus Tabelle 1 ergebenden Graphen zeigt Bild 5. Mithilfe des Graphen, der sich aus dem qualitativen Wissen über die Fehlerauswirkungen aufstellen lässt, kann die Regelbasis einfach hergeleitet werden.

Wie die letzten Betrachtungen schon nahelegen, verhalten sich das DRFS und ein entsprechend ausgelegter Zustandsautomat ähnlich [2]. Würden binäre Zuge-

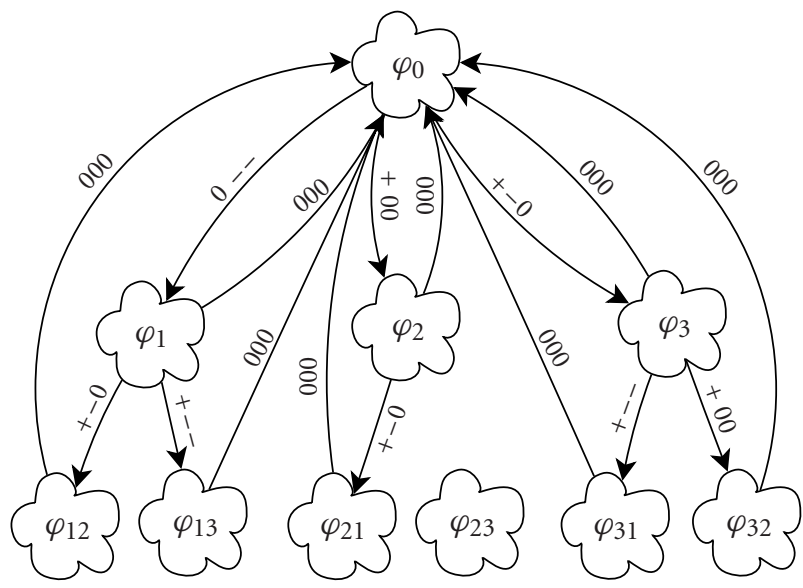

Bild 5 Darstellung der Fehlersymptomtabelle als linguistischer Zustandsautomat. Der Doppelfehler $\varphi_{23}$ ist nicht isolierbar.

hörigkeitsfunktionen verwendet, wären beide Systeme äquivalent. Das DRFS hat allerdings dann Vorteile, wenn die Symptome aufgrund von nicht modellierter Dynamik oder Rauschen von ihren nominellen Werten abweichen. Um dies zu illustrieren wird wieder die Symptomtabelle 1 betrachtet. Wir gehen davon aus, dass das Symptom $\rho_{2}$ bei Auftreten von Fehler $\varphi_{3}$ aufgrund von Modellierungsungenauigkeiten nicht so stark negativ wird wie erwartet. Die harte Grenze von „null“ nach „negativ“ wird dadurch beim Zustandsautomaten nicht überschritten, das DRFS befindet sich im Interpolationsbereich zwischen den Kernpositionen „negativ“ und „null“. Der Zustandsautomat wird aufgrund der Symptome „+ +00 “ auf den falschen Fehlerfall $\varphi_{2}$ schließen. Das DRFS dagegen wird aufgrund der unscharfen Grenzen auch Fehler $\varphi_{3}$ mit einer bestimmten Fehlerzugehörigkeit anzeigen. Tritt nun zusätzlich Fehler $\varphi_{1}$ auf, so kann der Zustandsautomat diesen nicht anzeigen, da nach der Erkennung von $\varphi_{2}$ der Doppelfehler $\varphi_{31}$ nicht erreicht werden kann (vgl. Bild 5). Da das DRFS den Zugehörigkeitswert zum Fehler $\varphi_{3}$ zurückführt, isoliert das DRFS den Doppelfehler $\varphi_{31}$ dagegen korrekt. Das DRFS ist so deutlich weniger anfällig gegenüber Störungen und Modellungenauigkeiten als der Zustandsautomat. Auch die Auslegung der Zugehörigkeiten ist dementsprechend einfacher. Obiges Verhalten wird anhand des Anwendungsbeispiels im folgenden Abschnitt nochmals betrachtet.

\section{Anwendungsbeispiel: Dreitank-System}

Der vorgestellte Ansatz zur FDI soll im Folgenden anhand des Dreitank-Benchmarksystems [20] erläutert werden, das in Bild 6 illustriert ist. Die Modellbildung des Systems erfolgt über die Bilanzgleichungen für die drei Tanks. Verwendet man die Füllstände $h_{i}$ als Zustandsgrößen $x_{i}$, ergibt sich das Zustandsraummodell

$A_{T} \dot{x}_{1}=Q_{z u}-Q_{12}$,

$A_{T} \dot{x}_{2}=Q_{12}-Q_{23}$,

$A_{T} \dot{x}_{3}=Q_{23}-Q_{a b}$, 


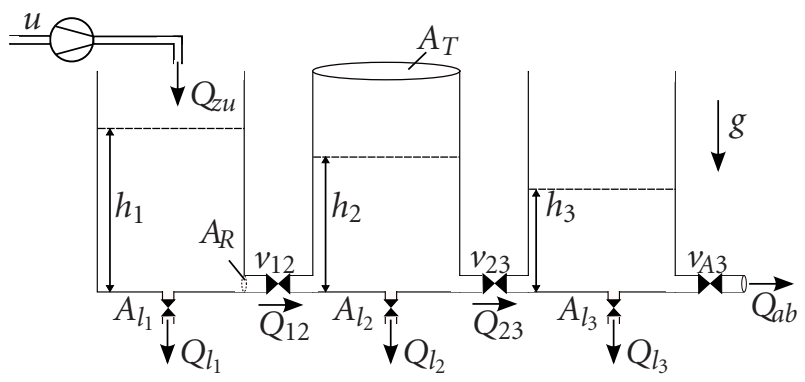

Bild 6 Schematische Darstellung des Dreitank-Systems einschließlich der Ventile zur Veranschaulichung der Fehlereinflüsse. Der Volumenstom $Q_{l}$ fließt im Falle eines Lecks der Größe $A_{l i}$ aus dem Tank $i \mathrm{ab}$. Eine Verstopfung des Ausmaßes $v_{\mu \nu}$ beeinflusst die zwischen den Tanks fließenden Volumenströme $Q_{\mu \nu}$.

mit den Volumenströmen

$Q_{\mu v}=c_{\mu v} A_{R_{\mu v}} \cdot \operatorname{sign}\left(x_{\mu}-x_{v}\right) \sqrt{2 g \cdot\left|x_{\mu}-x_{v}\right|}$

zwischen Tank $\mu$ und Tank $v$ bzw. dem Volumenstrom im Abfluss

$Q_{a b}=c_{a b} A_{R_{a b}} \sqrt{2 g \cdot x_{3}}$.

Hierbei sind $c_{\mu v}=1$ und $c_{a b}=0,8$ Durchflussparameter sowie $A_{T}=0,0157 \mathrm{~m}^{2}$ bzw. $A_{R_{\mu \nu}}=5 \times 10^{-5} \mathrm{~m}^{2}$ Tankbzw. Rohrquerschnitte.

Zur Validierung der vorgestellten Methoden zur FDI werden die in Bild 6 gezeigten Fehlerfälle untersucht. Hierbei handelt es sich zum einen um Leckagen $\left(\varphi_{1}, \ldots, \varphi_{3}\right)$ in den drei Tanks, die als zusätzliche Abflüsse

$Q_{l_{i}}=c_{a b} A_{l_{i}}(t) \sqrt{2 g \cdot x_{i}}$,

mit den zeitvarianten Leckquerschnitten $A_{l_{i}} \in\left[0, A_{R}\right)$ in den Bilanzgleichungen (16) modelliert werden. Zum anderen werden Verstopfungen in den Verbindungsrohren zwischen Tank 1 und Tank $2\left(\varphi_{4}\right)$ bzw. Tank 2 und Tank 3 $\left(\varphi_{5}\right)$ sowie im Abfluss $\left(\varphi_{6}\right)$ durch variierende Querschnittsflächen

$A_{R_{\mu v}}(t)=A_{R}\left(1-v_{\mu v}(t)\right) \quad$ mit $v_{\mu v}(t) \in[0,1)$

berücksichtigt.

Der erste Schritt zur FDI besteht in der Modellierung des Dreitank-Systems durch ein DRFS. Zur Generierung von Trainings- und Validierungsdaten wird das Modell mit einer Abtastzeit von $T=5 \mathrm{~s}$ diskretisiert und mit amplitudenmodulierten Pseudo-Rausch-Binär-Signalen angeregt. Mithilfe der so gewonnenen Datensätze wird nun ein DRFS aufgebaut, das das Dreitank-System sehr genau nachbilden kann. Die Residuen werden dann auf Basis dieses Modells und der Struktur aus Bild 4 bestimmt. Die Fehlersymptome $\rho_{i}$ werden schließlich gemäß

$\rho_{i}=\frac{r_{i}-r_{0, i}}{u}, \quad u>0$,

mit den Residuen $r_{0, i}$ im fehlerfreien Zustand gebildet. Durch die Normierung auf die Eingangsgröße $u$ reagieren die Symptome weniger empfindlich auf Schwankungen der Eingangsgröße. Als Ergebnis ergibt sich die Fehlersymptomtabelle 2, die alle Einzelfehler und Doppelfehler beinhaltet. Die mit Fragezeichen gekennzeichneten Einträge der Tabelle zeigen an, dass das Residuum bei Doppelfehlern abhängig von den jeweiligen Fehlergrößen sowohl positiv als auch negativ werden kann.

Betrachtet man nur die Einzelfehler, so zeigt sich, dass die Residuen aufgrund der FDI-Struktur nur sensitiv auf Fehler sind, die direkt auf den jeweiligen Zustand wirken. Entsprechend können alle Einzelfehler durch ein SFS isoliert werden. Weiterhin können aufgrund der Symptomverteilung auch einige Doppelfehler durch ein SFS isoliert werden. Problematisch ist dagegen beispielsweise der Doppelfehlerfall $\varphi_{24}$. Dieser hat dieselbe Symptomcharakteristik wie der Einzelfehler $\varphi_{4}$, sodass das SFS diesen Fall nicht isolieren kann. Verwendet man dagegen ein DRFS, so ist der Doppelfehler eindeutig isolierbar, wenn der Fehler $\varphi_{2}$ zuvor erkannt wurde. Tabelle 3 stellt die Isolierbarkeit der Einzel- und Doppelfehler für das SFS und DRFS zusammen.

Hierbei lassen sich verschiedene Klassen unterscheiden:

- Weiß hinterlegt: Diese Fehler sind sowohl mit dem SFS als auch mit dem DRFS isolierbar.

- Hellgrau hinterlegt: Diese Fehler sind nicht mit dem SFS, aber mit dem DRFS isolierbar.

- Dunkelgrau hinterlegt: Diese Fehler sind mit dem SFS nicht isolierbar. Die Isolierbarkeit mit dem DRFS hängt von den Fehlergrößen ab. Ist die Auswirkung des zweiten auftretenden Fehlers auf das Residuum deutlich kleiner als die des ersten, so ist der Doppelfehler nicht isolierbar. Der erste Fehler verdeckt den zweiten. Ist die Auswirkung des zweiten Fehlers hinreichend groß, ist der Doppelfehler dagegen isolierbar.

- Schwarz hinterlegt: Diese Fehler sind weder mit dem SFS noch mit dem DRFS isolierbar.

Insgesamt lassen sich 6 Einzelfehler sowie weitere 16 Doppelfehler mithilfe der Residuenstruktur nach Bild 4 bereits mit einem SFS isolieren. Durch die Verwendung eines

Tabelle 2 Fehlersymptomtabelle für das Dreitank-System. Als Fehlerfälle werden die Leckagen in den Tanks $\left(\varphi_{1}, \ldots, \varphi_{3}\right)$ und die Verstopfungen $\left(\varphi_{4}, \ldots, \varphi_{6}\right)$ sowie alle Doppelfehler $\varphi_{i j}$ betrachtet. Die Doppelfehler $\varphi_{j i}$ haben die gleichen Symptome wie $\varphi_{i j}$ und sind deshalb nicht explizit aufgeführt. $\varphi_{0}$ bezeichnet den fehlerfreien Zustand.

\begin{tabular}{|c|c|c|c|c|c|c|c|c|c|c|c|}
\hline & $\varphi_{0}$ & $\varphi_{1}$ & $\varphi_{2}$ & $\varphi_{3}$ & $\varphi_{4}$ & $\varphi_{5}$ & $\varphi_{6}$ & $\varphi_{12}$ & $\varphi_{13}$ & $\varphi_{14}$ & $\varphi_{15}$ \\
\hline$\rho_{1}$ & 0 & - & 0 & 0 & + & 0 & 0 & - & - & $?$ & - \\
\hline$\rho_{2}$ & 0 & 0 & - & 0 & - & + & 0 & - & 0 & - & + \\
\hline \multirow[t]{2}{*}{$\rho_{3}$} & 0 & 0 & 0 & - & 0 & - & + & 0 & - & 0 & - \\
\hline & $\varphi_{16}$ & $\varphi_{23}$ & $\varphi_{24}$ & $\varphi_{25}$ & $\varphi_{26}$ & $\varphi_{34}$ & $\varphi_{35}$ & $\varphi_{36}$ & $\varphi_{45}$ & $\varphi_{46}$ & $\varphi_{56}$ \\
\hline$\rho_{1}$ & - & 0 & + & 0 & 0 & + & 0 & 0 & + & + & 0 \\
\hline$\rho_{2}$ & 0 & - & - & $?$ & - & - & + & 0 & $?$ & - & + \\
\hline$\rho_{3}$ & + & - & 0 & - & + & - & - & $?$ & - & + & $?$ \\
\hline
\end{tabular}


Tabelle 3 Übersicht über die Isolierbarkeit der Fehlerfälle. 22 der 36 Fehlerfälle können durch ein SFS isoliert werden (weiß hinterlegt). Durch das DRFS können vier weitere vollständig (hellgrau) und sechs teilweise (grau) isoliert werden. Schwarz hinterlegte Fehlerfälle können nicht isoliert werden.

\begin{tabular}{lcccccc}
\hline \multicolumn{7}{c}{ 2. Fehler } \\
& $\varphi_{1}$ & $\varphi_{2}$ & $\varphi_{3}$ & $\varphi_{4}$ & $\varphi_{5}$ & $\varphi_{6}$ \\
\hline 1. Fehler & & & & & & \\
$\varphi_{1}$ & $\varphi_{1}$ & $\varphi_{12}$ & $\varphi_{13}$ & $\varphi_{14}$ & $\varphi_{15}$ & $\varphi_{16}$ \\
$\varphi_{2}$ & $\varphi_{21}$ & $\varphi_{2}$ & $\varphi_{23}$ & $\varphi_{24}$ & $\varphi_{25}$ & $\varphi_{26}$ \\
$\varphi_{3}$ & $\varphi_{31}$ & $\varphi_{32}$ & $\varphi_{3}$ & $\varphi_{34}$ & $\varphi_{35}$ & $\varphi_{36}$ \\
$\varphi_{4}$ & $\varphi_{41}$ & $\varphi_{42}$ & $\varphi_{43}$ & $\varphi_{4}$ & $\varphi_{45}$ & $\varphi_{46}$ \\
$\varphi_{5}$ & $\varphi_{51}$ & $\varphi_{52}$ & $\varphi_{53}$ & $\varphi_{54}$ & $\varphi_{5}$ & $\varphi_{56}$ \\
$\varphi_{6}$ & $\varphi_{61}$ & $\varphi_{62}$ & $\varphi_{63}$ & $\varphi_{64}$ & $\varphi_{65}$ & $\varphi_{6}$ \\
\hline
\end{tabular}

DRFS lassen sich vier weitere Fälle vollständig sowie sechs Fälle abhängig von der Fehlergröße isolieren.

Abschließend sollen zwei Beispielszenarien zur FDI gezeigt werden. Im ersten Szenario soll das DRFS mit einem SFS verglichen werden. Hierzu wird das Drei-TankSystem mit dem Eingangssignal aus Bild 7 beaufschlagt. Im zunächst fehlerfreien Betrieb tritt zum Zeitpunkt $t=50$ min Fehler $\varphi_{2}$, d. h. eine Leckage in Tank 2 auf. Nach Beseitigung dieses Fehlers tritt bei $t=150$ min wieder eine Leckage in Tank 2 auf. Zusätzlich verstopft bei $t=170$ min das Rohr zwischen Tank 1 und 2 (Fehler $\left.\varphi_{4}\right)$. Beide Fehler werden bei $t=200 \mathrm{~min}$ behoben. Dieser Vorgang wird zweimal mit zunehmenden Größen der Verstopfung wiederholt. Die Bilder 8 und 9 zeigen die Ergebnisse der FDI mit SFS und DRFS. Sowohl das SFS als auch das DRFS können den Einzelfehler $\varphi_{2}$ korrekt isolieren. Wie anhand der Fehlersymptomtabelle zu erwarten, kann das SFS den Doppelfehlerfall $\varphi_{24}$ allerdings nicht isolieren. Vielmehr wird fälschlicherweise der Doppelfehler $\varphi_{34}$ angezeigt. Das DRFS kann dagegen unabhängig von den Fehlergrößen die jeweiligen Fehlerfälle richtig isolieren.

Im zweiten Szenario wird das DRFS mit einem Zustandsautomaten verglichen. Letzterer wurde so ausgelegt, dass die harten Zustandsübergänge auf dem Mittelwert der zwei zugehörigen Kernpositionen des DRFS liegen. Im Szenario tritt zum Zeitpunkt $t=20 \mathrm{~min}$ Fehler $\varphi_{5}$ mit einer Verstopfung von $5 \%$ zwischen Tank 2 und 3 auf. Aufgrund der sehr kleinen Fehlergröße wird das Symptom $\rho_{2}$ weniger stark positiv als angenommen.

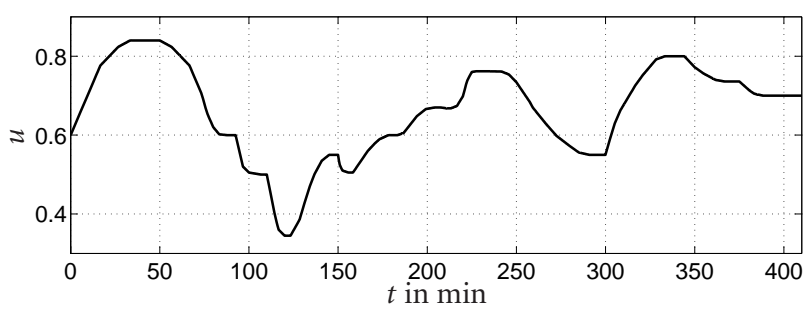

Bild 7 Anregungssignal für den Dreitank-Prozess.
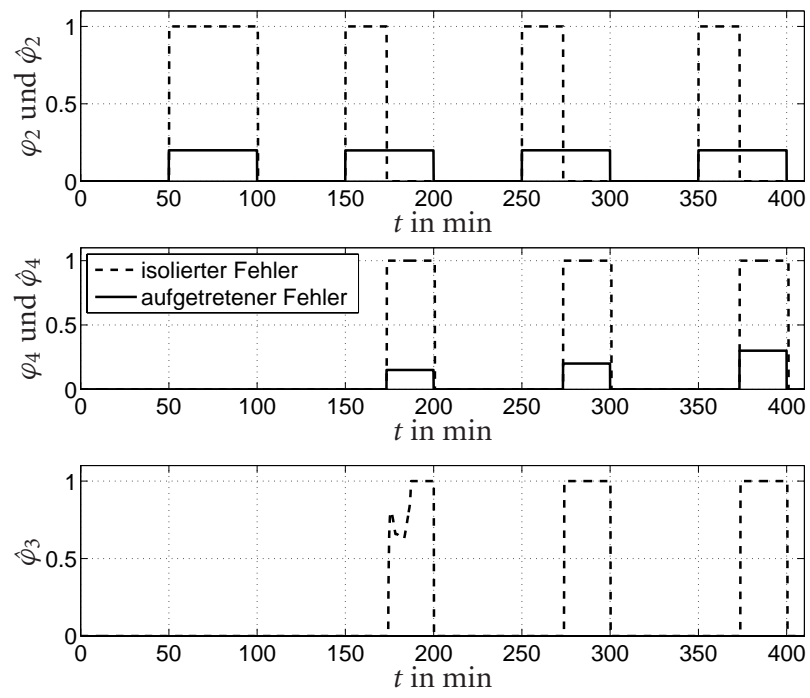

Bild 8 Ergebnisse der Fehlerisolation mit SFS. Dargestellt sind die Komponenten des isolierten Fehlervektors $\hat{\varphi}$ (strichliert) und die tatsächlich auftretenden Fehler $\boldsymbol{\varphi}$ (durchgezogen). Der Fehler $\hat{\varphi}_{3}$ wird fälschlicherweise erkannt.
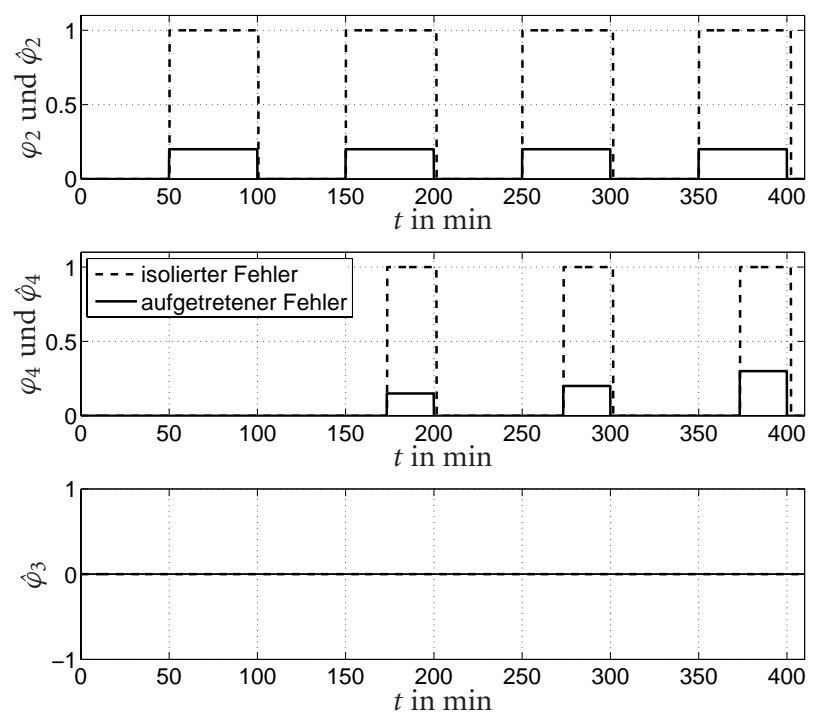

Bild 9 Ergebnisse der Fehlerisolation mit DRFS. Dargestellt sind die Komponenten des isolierten Fehlervektors $\hat{\varphi}$ (strichliert) und die tatsächlich auftretenden Fehler $\varphi$ (durchgezogen). Alle Fehlerfälle werden korrekt isoliert.

Zum Zeitpunkt $t=80$ min verstopft dann zusätzlich der Abfluss (Fehler $\varphi_{6}$ ). Die Bilder 10 und 11 zeigen die Ergebnisse für den Zustandsautomaten und das DRFS. Aufgrund der auftretenden Symptome erkennt der $\mathrm{Zu}$ standsautomat fälschlicherweise Fehler $\varphi_{3}$ während das DRFS beide möglichen Fehler $\varphi_{3}$ und $\varphi_{5}$ mit den Fehlerzugehörigkeiten 0,55 bzw. 0,45 erkennt. Tritt nun der zweite Fehler auf, wird der Doppelfehler $\varphi_{56}$ vom DRFS mit Wahrscheinlichkeit 1 richtig isoliert. Der Zustandsautomat wechselt dagegen zunächst in den fehlerfreien Zustand und isoliert dann lediglich den Fehler $\varphi_{6}$. Der Fehler $\varphi_{5}$ wird gar nicht erkannt. Dies veranschaulicht die größere Robustheit DRFS im Vergleich zu Zustandsauto- 

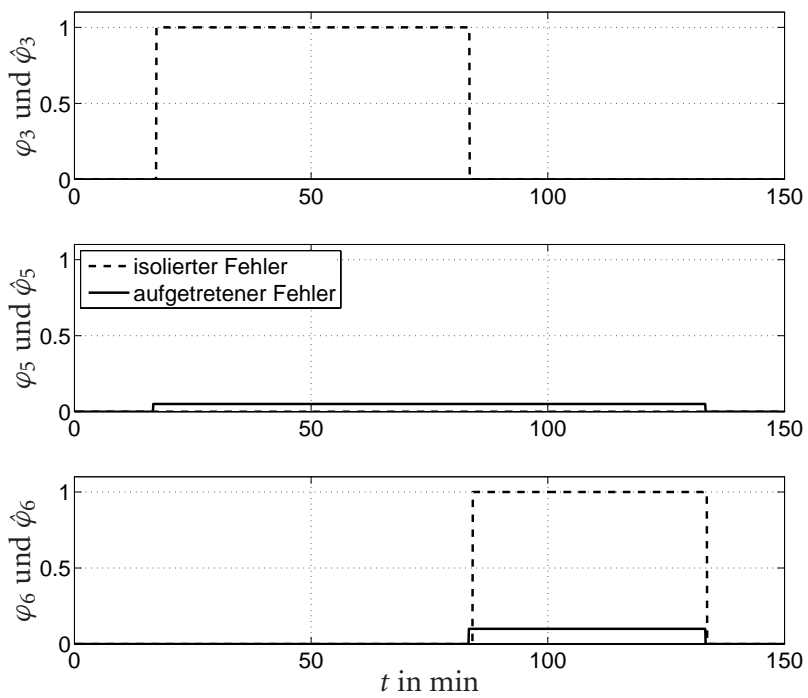

Bild 10 Ergebnisse der Fehlerisolation mit einem Zustandsautomat. Zunächst wird fälschlicherweise $\varphi_{3}$ erkannt. Beim zusätzlichen Auftreten von Fehler $\varphi_{6}$ wird zunächst kein Fehler und dann Fehler $\varphi_{6}$ erkannt. Der Fehler $\varphi_{5}$ wird dagegen gar nicht erkannt.
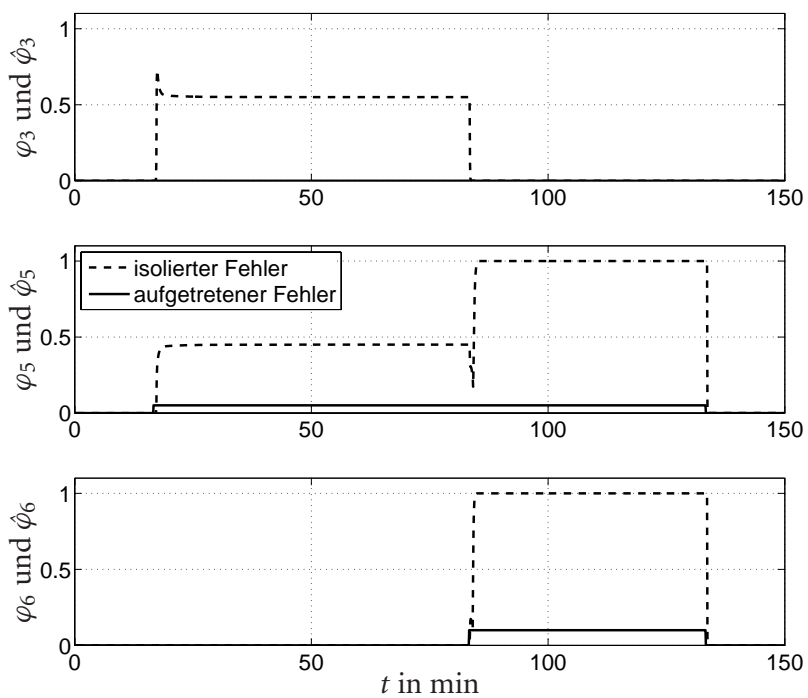

Bild 11 Ergebnisse der Fehlerisolation mit DRFS. Zunächst werden die Fehler $\varphi_{3}$ und $\varphi_{5}$ mit den Fehlerzugehörigkeiten 0,55 bzw. 0,45 erkannt. Bei Auftreten von Fehler $\varphi_{6}$ wird der Doppelfehler $\varphi_{56}$ korrekt isoliert.

maten bei Modellungenauigkeiten wie der beschriebenen geringeren Fehlergröße.

\section{Zusammenfassung}

Dieser Beitrag zeigt die Anwendung zeitdiskreter rekurrenter Fuzzy-Systeme zur Fehlerdetektion und -isolation. Im Bereich der Detektion wurde eine Möglichkeit vorgestellt, wie man auf Basis trainierter nichtlinearer $\mathrm{Zu}$ standsraummodelle sensitive Residuen generieren kann. Durch die Verwendung DRFS zur Modellierung des Prozesses kann über die reine datengestützte Modellbildung hinaus Expertenwissen in Form von Regeln in den Modellbildungsprozess eingebracht werden. Außerdem wurde ausgehend von statischen Fuzzy-Systemen zur
Fehlerisolation eine Erweiterung auf DRFS vorgestellt, die insbesondere beim Auftreten mehrerer aufeinander folgender Fehler eine genauere Fehlerisolation ermöglicht. Die Anwendung am Dreitank-System illustriert dies.

Für den Einsatz DRFS zur Fehlerdetektion stehen leistungsfähige Lernverfahren zur Verfügung. Im Gegensatz dazu erfolgt die Auslegung DRFS zur Mehrfehlerisolation, insbesondere die Einstellung der Regeln, bislang noch heuristisch mittels Erfahrungswissen. Aufgrund der relativ großen Regelbasis kann dies zeitaufwendig sein. Als Erweiterung des bisherigen Ansatz bietet es sich deshalb an, das DRFS auf Basis zur Verfügung stehender repräsentativer Datensätze der Fehlerfälle zu trainieren und so den Entwurfsprozess zu automatisieren.

\section{Literatur}

[1] AdAmy, J.: Device for early detection of runout in continuous casting. - EP Priority Date 03.04.1995, European Patent 0819033B1 (1998), US Patent 5,904,202 (1999).

[2] Adamy, J.; Kempf, R.: Regularity and chaos in recurrent fuzzy systems. In: Fuzzy Sets and Systems 140(2) (2003), S. 259-284.

[3] Adamy, J.; Schwung, A.: Modellierung dynamischer Systeme mit zeitkontinuierlichen rekurrenten Fuzzy-Systemen. In: Automatisierungstechnik 57(3) (2009), S. 129-137.

[4] Blanke, M.; Kinnaert, M.; Lunze, J.; Staroswiecki, M.: Diagnosis and Fault-Tolerant Control. Springer, 2006.

[5] De Kleer, J.; Williams, B.: Diagnosing multiple faults. In: Artificial Intelligence 32(1) (1987), S. 97-130.

[6] Flemming, A.; Kempf, R.; Adamy, J.: Traffic simulation employing recurrent fuzzy systems. In: Proc. of the 16th IASTED Intern. Conf. Applied Simulation and Modelling, 2007, S. 497-502.

[7] Frank, P.: Fault Diagnosis in Dynamic Systems Using Analytical and Knowledge-based Redundancy - A Survey and Some New Results. In: Automatica 26 (1990), S. 459-474.

[8] Frank, P.; Köppen-Seliger, B.: Fuzzy Logic and Neural Network Applications to Fault Diagnosis. In: International Journal of Approximate Reasoning 16 (1997), S. 67-88.

[9] Gertler, J.: Fault detection and isolation using parity relations. In: Control Engineering Practice 5(5) (1997), S. 653-661.

[10] Gert Ler, J.; Singer, D.: A new structural framework for parity equation-based failure detection and isolation. In: Automatica 26(2) (1990), S. 381-388

[11] Gorrini, V.; Bersini, H.: Recurrent fuzzy systems. In: Proc. 3rd IEEE Conf. on Fuzzy Systems Bd. 1. S. 193-198, Orlando, USA, 1994.

[12] Isermann, R.: Process fault detection based on modeling and estimation methods - A survey. In: Automatica 20(4) (1984), S. 387-404.

[13] Is ermann, R.: Fault Diagnosis Systems - An Introduction from Fault Detection to Fault Tolerance. Springer, 2006.

[14] Kempf, R.: Rekurrente Fuzzy-Systeme. Fortschrittberichte VDI, Reihe 8, Nr. 1028, VDI Verlag, Düsseldorf, Universität Darmstadt, Diss., 2004.

[15] KEMPF, R.; A DAmy, J.: Equilibria of recurrent fuzzy systems. In: Fuzzy Sets and Systems 140(2) (2003), S. 231-257.

[16] Kempf, R.; Adamy, J.: Sequential pattern recognition employing recurrent fuzzy systems. In: Fuzzy Sets and Systems 146(3) (2004), S. 451-472.

[17] Leonhardt, S.; Ayoubi, M.: Methods of Fault Diagnosis. In: Control Engineering Practice 5(5) (1997), S. 683-692.

[18] Lunze, J.: Ereignisdiskrete Systeme. Oldenbourg Verlag, 2006.

[19] NyberG, M.: A fault isolation algorithm for the case of multiple faults and multiple fault types. In: Proc. of the14th IFAC Symposium on System Identification. Newcastle, Australien, 2006. 
[20] Рatton, R.; Chen, J.; Siew, T.: Fault Diagnosis in Nonlinear Systems via Neural Networks. In: Proc. of the IEEE Intern. Conf. on Control. Coventry, Großbritannien, 1994.

[21] Patton, R.; Uppal, F.; Lopez Toribio, C.: Soft Computing approaches to fault diagnosis for dynamic systems: A survey. In: Proc. of 4th IFAC Symposium on Fault Detection Supervision and Safety for Technical Processes. Budapest, 2000.

Manuskripteingang: 8. September 2009

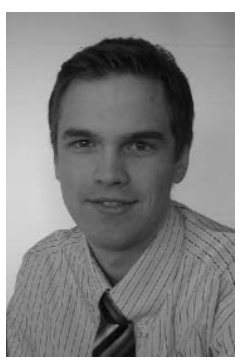

Dipl. Ing. Andreas Schwung ist wissenschaftlicher Mitarbeiter am Fachgebiet Regelungstheorie und Robotik im Fachbereich Elektrotechnik und Informationstechnik der Technischen Universität Darmstadt. Hauptarbeitsgebiete: Modellbildung dynamischer Systeme, Fehlerdiagnose, Rekurrente Fuzzy-Systeme.

Adresse: Technische Universität Darmstadt, Fachbereich Elektrotechnik und Informationstechnik, FG Regelungstheorie und Robotik, 64283 Darmstadt, Fax: +49-(0)6151-16-2507,

E-Mail: aschwung@rtr.tu-darmstadt.de

Verfügbar unter

lediglich die vom Gesetz vorgesehenen Nutzungsrechte gemäß UrhG

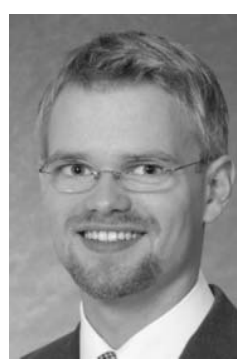

Dipl. Ing. Andreas Ortseifen ist wissenschaftlicher Mitarbeiter am Fachgebiet Regelungstheorie und Robotik im Fachbereich Elektrotechnik und Informationstechnik der Technischen Universität Darmstadt. Hauptarbeitsgebiete: Nichtlineare Regelungen, Anti-Windup.

Adresse: Technische Universität Darmstadt, Fachbereich Elektrotechnik und Informationstechnik, FG Regelungstheorie und Robotik, 64283 Darmstadt, Fax: +49-(0)6151-16-2507,

E-Mail: ortseifen@rtr.tu-darmstadt.de

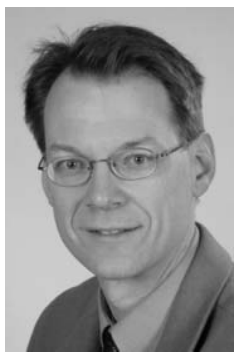

Prof. Dr.-Ing. Jürgen Adamy ist Leiter des Fachgebiets Regelungstheorie und Robotik im Fachbereich Elektrotechnik und Informationstechnik der Technischen Universität Darmstadt. Hauptarbeitsgebiete: Regelungsverfahren, Computational Intelligence, autonome mobile Roboter.

Adresse: Technische Universität Darmstadt, Fachbereich Elektrotechnik und Informationstechnik, Fachgebiet Regelungstheorie und Robotik, Landgraf-Georg-Str. 4, 64283 Darmstadt, Fax: +49-(0)6151-16-2507,

E-Mail: adamy@rtr.tu-darmstadt.de 